\title{
Safety and Short-Term Efficacy of Irreversible Electroporation and Allogenic Natural Killer Cell Immunotherapy Combination in the Treatment of Patients with Unresectable Primary Liver Cancer
}

\author{
Yumei Yang ${ }^{1} \cdot$ Zilin Qin $^{2} \cdot$ Duanming Du ${ }^{1}$ (I) Yumin $\mathrm{Wu}^{1} \cdot$ Shuibo Qiu ${ }^{1}$. \\ Feng $\mathrm{Mu}^{4} \cdot$ Kengcheng $\mathrm{Xu}^{5} \cdot$ Jibing Chen ${ }^{3,5}$
}

Received: 20 April 2018/Accepted: 21 August 2018/Published online: 27 August 2018

(C) The Author(s) 2018

\begin{abstract}
Purpose This study aimed to investigate the safety and short-term efficacy of irreversible electroporation (IRE) combined with allogenic natural killer (NK) cell immunotherapy in the treatment of patients with unresectable primary liver cancer.

Materials and Methods Between October 2015 and December 2016, 40 patients were enrolled and randomly allocated to either the IRE group $(n=22)$ or the IRE-NK group $(n=18)$. All adverse events experienced by the patients were recorded; the changes in tumor biomarkers [AFP, CA 19-9, circulating tumor cells (CTCs)], lymphocyte number and function, quality of life, clinical response, progression-free survival (PFS) and overall survival (OS) were assessed.
\end{abstract}

Electronic supplementary material The online version of this article (https://doi.org/10.1007/s00270-018-2069-y) contains supplementary material, which is available to authorized users.

Duanming Du

dmdu69@163.com

$\triangle$ Jibing Chen

jibingchen398@163.com

1 Department of Interventional Therapy, The First Affiliated Hospital of Shenzhen University, Shenzhen Second People's Hospital, No. 3002 of SunGang West Road, FuTian, Shenzhen 518035, China

2 Chongqing Health Service Center, Chongqing 400020, China

3 Biotherapy Center, Fuda Cancer Hospital of Jinan University, Guangzhou 510665, China

4 Department of Oncology, Fuda Cancer Hospital of Jinan University, Guangzhou, China

5 Fuda Cancer Institute, Guangzhou, China
Results Patients who received combination therapy exhibited significantly longer median PFS and OS than who just received IRE (PFS 15.1 vs. 10.6 months, $P<0.05$, OS 17.9 vs. 23.2 months, $P<0.05)$. The combination therapy of IRE and NK cell immunotherapy significantly reduced CTCs and increased immune function and Karnofsky performance status.

Conclusion Our data suggest a novel, promising combination therapy using IRE and allogenic NK cell immunotherapy. Larger clinical trials are required to confirm these conclusions.

Keywords Irreversible electroporation · Natural killer cells · Primary liver cancer · Immunotherapy · Clinical trial

\section{Introduction}

Primary liver cancer (PLC) is the second leading cause of cancer deaths in less developed countries and is the sixth leading cause of cancer deaths among men in developed countries. Its incidence has been increasing every year, with China alone accounting for about $50 \%$ of the total estimated new liver cancer cases $[1,2]$. Surgery, although regarded as the gold standard treatment, is suitable for less than $20 \%$ of patients with PLC due to multiple tumors, metastasis, hepatic function compromise, or other deleterious factors [3]. Most PLCs are unresectable when diagnosed. Transcatheter arterial chemoembolization (TACE) and sorafenib treatment have shown promise in randomized controlled trials in selected unresectable PLC 
populations [4-6]. However, TACE-related toxicity has limited its use to intermediate stage PLC [7], and sorafenib extends the overall survival (OS) by only 3 months. Subsequent efforts in drug developments have failed [8]. Percutaneous thermal ablation is considered the optimal treatment choice for focal unresectable PLC of early stage; however, there is a risk of collateral thermal damage to sensitive adjacent organs: when the target lesion is adjacent to vessel, the heat-sink effect can cause the ablation of the lesion to be incomplete, the shape and size of the ablation zone may be unpredictable [9]. This challenging clinical scenario warrants new, safe, effective, and life-prolonging strategies for patients with PLCs.

In recent years, irreversible electroporation (IRE), a new, nonthermal and minimally invasive technique developed on the basis of reversible electroporation technology, has been used increasingly in the clinic, which is not affected by the heat-sink or cold-sink effect that may lead to incomplete ablation especially in perivascular tumor cells, and causing little damage to normal tissues, such as the gall bladder, bile duct [10-12]. Therefore, IRE permits the treatment of tumors unsuitable for surgical resection or thermal therapies. Moreover, the large number of tumorspecific antigen remaining in situ after IRE lacks thermal denaturation, resulting in a more potent immune response than thermal ablation which enhances the therapeutic outcome. Many studies have shown immunocyte infiltration in ablated areas following IRE treatment [13-15]. Studies in rats have suggested that IRE treatment changes the status of cellular immunity with a significant increase in peripheral lymphocytes and serum cytokines [16]. But the recurrence of PLCs following IRE remains a common phenomenon in the clinical setting [17].

The clinical application of natural killer (NK) cell-based immunotherapy is in its initial stage, with allogenic NK cells increasingly pursued for adoptive cellular therapies since the discovery of the renowned killer immunoglobulin-like receptor (KIR)/histocompatibility antigens (HLA) mismatch [18]. Our previous research demonstrated the safety and efficacy of NK cell-based immunotherapy in the treatment of PLC patients [19]. IRE combined with allogenic NK cell immunotherapy has been applied to the treatment of pancreatic cancer and has proved to be safe and efficacious [20]. However, the combination therapy has not been applied to the treatment of PLC. NK cells are enriched in human livers, forming $30-50 \%$ of the intrahepatic lymphocytes. The immune surveillance exerted by NK cells is crucial to the immune functions and defense of the liver against cancer [21]. On this basis, we surmise that the IRE with allogenic NK cell immunotherapy may be a promising combination therapy for PLC.

This is the first prospective study which assessed the safety and short-term efficacy of IRE and allogenic NK cell
Fig. 1 A Study design flowchart. A total of 40 patients were included and stratified by center. Patients were allocated to groups by a permuted blocks randomization protocol. One patient allocated to the IRE-NK group rejected NK immunotherapy following IRE since failing to find out a KIR ligand donor and was thus excluded from the IRE-NK group $(n=18)$ and included in IRE group $(n=22)$. B Treatment schedule. Peripheral blood was collected to obtain the NK cells 1-4 days prior to IRE treatment. NK cell immunotherapy was started within 14 days following blood collection and given for 3 consecutive days (8-12 days after IRE). The next course of collection started 1 day before the last infusion of the previous course

immunotherapy combination in the treatment of patients with unresectable PLCs.

\section{Materials and Methods}

\section{Patients}

This prospective study enrolled 40 patients with PLC between October 2015 and December 2016. The inclusion criteria were as follows: (1) 20-80 years old; (2) clear diagnosis of PLC based on imaging and pathological findings with tumor lesion $<10 \mathrm{~cm}$; (3) unresectable PLC, which was defined as the impossibility of completely removing the tumor or retaining a sufficient liver remnant to maintain liver function; (4) liver function classified as Child-Pugh class A or B; (5) not more than 3 intrahepatic lesions or 3 extrahepatic metastatic lesions, no invasion of the portal vein, the hepatic vein trunk or secondary branches, and expected survival > 6 months; and (6) Karnofsky performance status (KPS) $>60$. Exclusion criteria: (1) serious abnormalities in liver, lung, heart or kidney function; (2) massive ascites or brain metastasis; (3) acute or chronic infection; (4) patients who had blood coagulation disorders, severe anemia, or other primary tumors and (5) patients who were positive for HIV, HTLV-1, syphilis, tuberculosis, or parasitic blood infections. The primary endpoint of the current study was progression-free survival (PFS) and OS of the treated lesions, as there have been no studies of direct comparison of IRE and IRE-NK regarding PFS or OS in the treatment of patients with unresectable PLC in a randomized clinical trial format. In our institution, approximately 75 patients with PLC were treated by IRE annually. We assumed that among those patients, 38 patients could be enrolled in this study. And assuming the drop rate as 5\%, size of the target population was determined as 40 . The patients were allocated to groups by stratified permuted blocks randomization method on gender and Child-Pugh scores (Child-Pugh A and Child-Pugh A) (Fig. 1A). The enrolled patients were allocated to either the IRE or the IRE-NK group. This study was approved by the Institutional Review Board of 


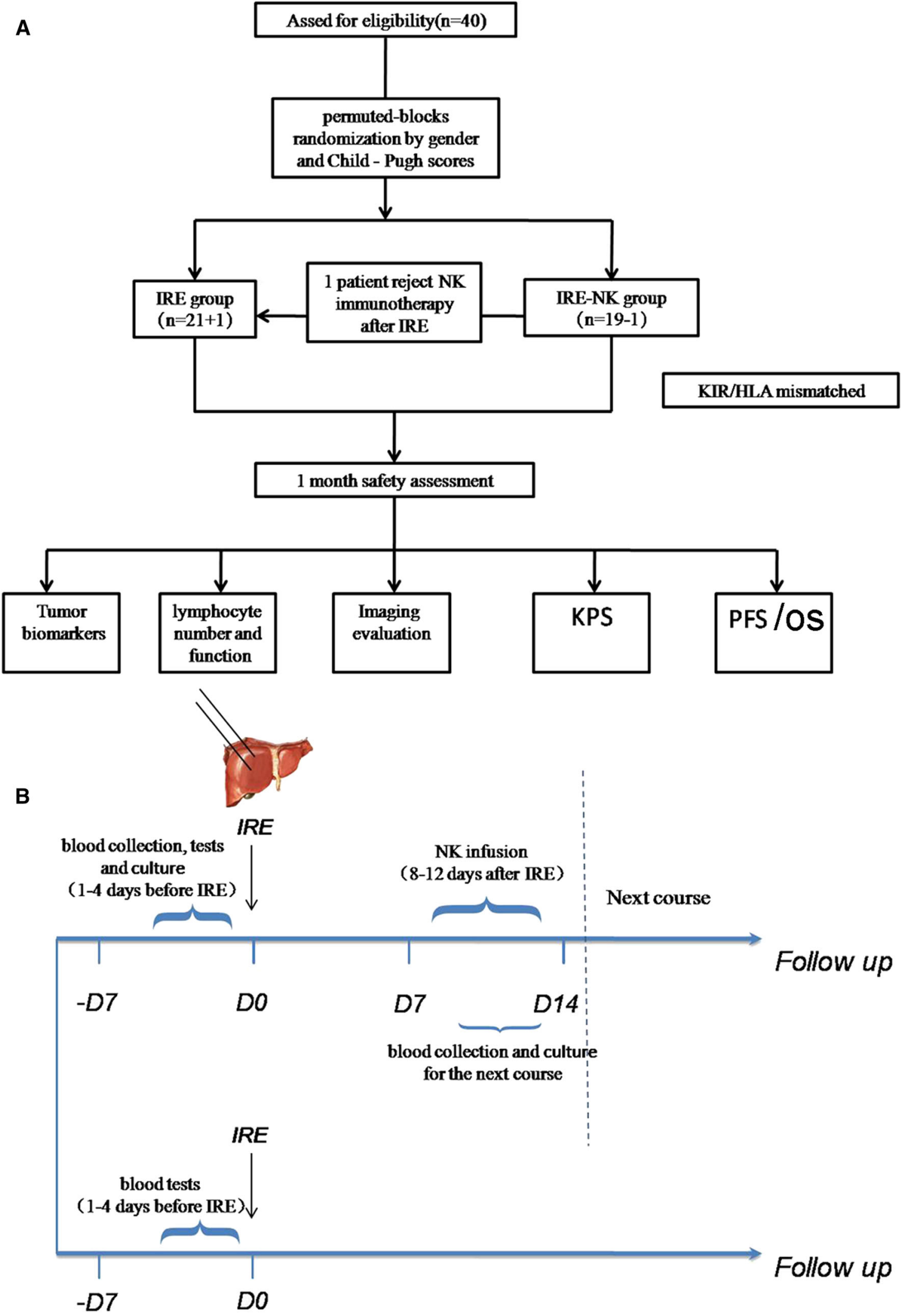


Fuda Cancer Hospital and complies with the provisions of the Declaration of Helsinki. All patients provided written informed consent. This trial was registered at ClinicalTrials.gov (Trial No. NCT03008343).

\section{Irreversible Electroporation Procedure}

All patients underwent muscle relaxants and general anesthesia. IRE was performed using an IRE ablation system (NanoKnife ${ }^{\mathrm{TM}}$ system, model HVP01; AngioDynamics, Queensbury, New York, USA). The main configuration includes a high-voltage current generator (maximum power output of $3 \mathrm{kV}, 50 \mathrm{~A}$ ), an electrocardiogram (ECG) synchronization (an AccuSyne ${ }^{\mathrm{R}}$ synchronizer Device, AccuSync Medical Research Corporation, Milford, Connecticut, USA), a $15-\mathrm{cm}$ pulse start probe (Model 20400103) and a 15-cm pulse standard probe (Model 20400104). All the percutaneous ablations were guided by computed tomography (CT SOMATOM Definition 64 AS; Siemens Medical Solutions, Forchheim, Germany) combined with ultrasound (US; IU22; Philips Medical Systems, Bothell). The number of probes, the method of inserting the needle, and the operation parameters were determined by the pre-operation plan of IRE. The distance between the electrodes was $1.5-2.5 \mathrm{~cm}$ [22], and the effective exposure probe distance was $1.5-2.5 \mathrm{~cm}$. The parameters of the IRE generator were set as follows: the pulse length was 70-90 $\mu$ s, the pulse repetition was $70-90$, and the average electric field intensity was $800-2200 \mathrm{v} / \mathrm{cm}$. One or more pullbacks were performed if the target region was $>2 \mathrm{~cm}$ in diameter. All pulses were delivered during the ventricular refractory period to avoid the occurrence of arrhythmias. Following the ablation, CT imaging was performed to confirm the ablation range was more than $0.5-1 \mathrm{~cm}$ on the edge of the tumor; the patient was then transferred to the intensive care unit for $24 \mathrm{~h}$, and then transferred to the general ward if their vital signs were stable. Relevant treatment was administered if there were any complications. Two surgeons with 4-8 years of experience in image-guided tumor ablation performed all procedures.

\section{IRE-NK Therapy Procedure}

The relatives of the enrolled patients were informed, and their peripheral blood was collected from KIR/HLA-mismatched donor to obtain the NK cells 1-4 days prior to IRE treatment [21]. The human high activity NK cell in vitro preparation kit (Hank Bioengineering Co. Ltd, Shenzhen, China) was used to expand and activate NK cells from peripheral blood mononuclear cells in vitro to prepare NK cells of higher quantity, purity, and activation, namely highly activated NK (HANK) cells (Electronic supplementary material [ESM] Fig. 1). NK cell immunotherapy was started within 14 days following blood collection and given for 3 consecutive days (8-12 days following IRE). The next course of collection started 1 day before the last infusion of the pervious course. The 18 patients in the IRE-NK group received continuous treatment of 4 courses of NK cell immunotherapy (Fig. 1B).

\section{Adverse Events}

Complications during treatment and post-treatment were evaluated in accordance with the Common Terminology Criteria of Adverse Events, v4.0.

\section{Tumor Biomarkers}

Circulating tumor cells (CTCs), serum $\alpha$-fetoprotein (AFP) and CA 19-9 were detected at 1-4 days pre-treatment and at 1 month post-treatment. AFP normal range: 0-5.8 IU/ ml, CA 19-9 normal range: 0-39 U/ml. CTCs was defined as CD45-negative, CK-positive, and CD326-positive cells and was quantified by flow cytometry (FACSCanto ${ }^{\mathrm{TM}} \mathrm{II}$; $\mathrm{BD}$, Grand Island, NY, USA).

\section{Imaging}

Dynamic CT/magnetic resonance imaging (MRI) abdominal scans were obtained prior to IRE after 1 month and then at 3 months post-treatment. The modified response evaluation criteria in solid tumors (mRECIST) was used to assess the tumors. To accurately observe the therapeutic effects, the total area of all tumors before and after treatment was compared. According to the criteria, clinical effects were divided into complete response (CR), partial response (PR), stable disease (SD), and progressive disease (PD). PFS was calculated from the first treatment to the time at which patients were found to meet the criteria for PD. CR + PR represent the effective rate (RR). The results were evaluated independently by 2 experienced radiologists, who were blinded to all other patient history. In the case of disagreement, they collectively read the film and reached a consensus.

\section{Karnofsky Performance Status}

KPS evaluation was recorded before and after treatment as an index of quality of life (QOL).

\section{Detection of Immune Function}

One to four days prior to IRE treatment, and 1 month following the final NK cell transfusion, peripheral blood 
(2 ml) was collected and assessed using flow cytometry (FACSCanto $^{\mathrm{TM}} \mathrm{II}$; BD, Grand Island, NY, USA). The tested indices included lymphocyte number and function in the patients' peripheral blood. A BD Multitest 6-color TBNK reagent (No. 644611) was used to detect the number of $\mathrm{CD} 3+\mathrm{CD} 4+$ cells, $\mathrm{CD} 3+\mathrm{CD} 8+$ cells, total CD3 + cells, CD3 - CD19+ cells, and CD3 CD16 + CD56 + cells. The BD cytometric bead array (CBA) human Th1/Th2 cytokine kit II (No. 551809) was used to detect the expression levels of interleukin-2 (IL-2), IL-4, IL-6, IL-10, tumor necrosis factor (TNF), and interferon- $\gamma($ IFN- $\gamma)$. The tests were performed according to the protocols in the instruction manuals. Results above or within the reference range were defined as normal; results below the reference range were defined as immune dysfunction. For the IRE group, peripheral blood was drawn 1-4 days prior to IRE and 1 month following IRE; blood was drawn from the IRE-NK group 1-4 days prior to IRE and 1 month following NK cell therapy.

\section{Statistical Analysis}

The primary end point of the study was PFS and OS; PFS was calculated from the date protocol treatment was started until the date of death or of the first evidence of radiographic disease progression. OS was calculated from the date the treatment was started until the date of death. Differences were considered significant at $P<0.05$. The basic characteristics, adverse events, and RR of the two groups were compared using the Chi-square test; immunity detection, tumor biomarkers, and KPS data were presented as the mean \pm standard deviation and compared using the Student's $t$-test. SPSS version 13.0 (IBM; Armonk, NY, USA) was used for the statistical analyses, and measurement data were expressed as the mean \pm standard deviation. GraphPad Prism 5 (GraphPad Software, San Diego, CA) was used to plot graphs and analyze the PFS and OS rate.

\section{Results}

\section{Patient Characteristics}

A total of 40 patients were included and randomly assigned to the IRE group $(n=21)$ or the IRE-NK group $(n=19)$. One patient allocated to the IRE-NK group rejected NK immunotherapy following IRE since failing to find out a KIR ligand donor and was excluded from IRE-NK group ( $n=18$ ). The patient was then included in the IRE group $(n=22)$. Patient demographics were not statistically different between the two groups (Table 1).

\section{Progression-Free Survival and Overall Survival}

The median PFS of patients who underwent IRE was 10.6 months, which is shorter than that of patients who underwent IRE combined with allogenic NK cell immunotherapy (15.1 months). The PFS was significantly different between these two groups $(P=0.018)$. One-year OS for IRE group and IRE-NK group was 66.7 and $77.8 \%$, respectively, with median OS 17.9 versus $23.2(P=0.031)$. However, OS of HCC or ICC in the interior group and inter-group was not significantly different (Fig. 2).

\section{Response to Treatment}

Treatment response was evaluated according to the mRECIST guidelines by 2 experienced radiologists. The interobserver reproducibility between readers 1 and 2 was almost in perfect agreement $(\kappa=0.858)$. The intra-observer reproducibility based on reader 1 's twice was almost in perfect agreement $(\kappa=0.903)$. Therefore, all outcomes were based on the measurements taken by the first radiologist. After follow-ups for 3 months, 1 patient in the IRE group and 3 patients in IRE-NK group displayed CR; PR was recorded in 14 patients of the IRE group and 13 patients in the IRE-NK group. Development of new lesions was observed in 1 patient from the IRE group at 2.4 months post-treatment using a contrast-enhanced CT scan. The RR in the IRE-NK group $(88.9 \%)$ was higher than in the IRE group $(68.2 \%)(P=0.15)$. The representative results are shown in Table 2 .

\section{Comparison of Immune Function}

In IRE group, the absolute number of lymphocyte subsets, Th1 cytokine (IL-2, IFN- $\beta$, IFN- $\gamma$ ) levels and IL-6 1 month post-treatment were higher than that before treatment. A similar and more obvious variation tendency was observed in IRE-NK group except IL-6. No significant changes in IL-4 or IL-10 were observed in both groups (Electronic supplementary material [ESM] Table 1).There were no significant differences in the lymphocyte count or Th1 cytokine levels between the two groups pre-treatment $(P>0.05)$,but which were higher in the IRE-NK group compared to the IRE group post-treatment $(P<0.05$, (Figure 3).

\section{Safety Evaluation}

The adverse events measured post-treatment include pain, pleural effusion, ascites, fatigue, and fever. The IRE group had $6,1,3,3$, and 10 patients with these symptoms, respectively. In the IRE-NK group, 4, 4, 4, 5, and 11 patients, respectively, exhibited these symptoms (ESM 
Table 1 Patient characteristics

\begin{tabular}{|c|c|c|c|}
\hline Factor & $\begin{array}{l}\text { IRE } \\
(n=22)\end{array}$ & $\begin{array}{l}\text { IRE-NK } \\
(n=18)\end{array}$ & $P$ value \\
\hline Gender & & & $P=0.676$ \\
\hline Male & 12 & 11 & \\
\hline Female & 10 & 7 & \\
\hline Median age (years) & 54 & 57 & $P=0.723$ \\
\hline Child-Pugh stratification & & & $P=0.822$ \\
\hline Class A & 9 & 8 & \\
\hline Class B & 13 & 10 & \\
\hline Clinical stage (AJCC) & & & $P=0.737$ \\
\hline III & 8 & 5 & \\
\hline IV & 14 & 13 & \\
\hline Histology & & & $P=0.750$ \\
\hline $\mathrm{HCC}$ & 13 & 9 & \\
\hline ICC & 9 & 9 & \\
\hline Tumor number & & & $P=0.286$ \\
\hline Solitary & 8 & 3 & \\
\hline Multifocal & 14 & 15 & \\
\hline $\begin{array}{l}\text { Maximal diameter largest } \\
\text { tumor }(\mathrm{cm})\end{array}$ & $4.73 \pm 1.62$ & $4.81 \pm 1.61$ & $P=0.871$ \\
\hline \multicolumn{4}{|l|}{ Mean \pm SD } \\
\hline $\begin{array}{l}\text { Maximal diameter largest } \\
\text { tumor }(\mathrm{cm})\end{array}$ & & & $P=0.523$ \\
\hline$<5$ & 14 & 9 & \\
\hline $5-10$ & 8 & 9 & \\
\hline AFP (IU/ml) & & & $P=0.6389$ \\
\hline$<200$ & 6 & 6 & \\
\hline $200-400$ & 2 & 3 & \\
\hline$\geq 400$ & 14 & 9 & \\
\hline KPS score & & & $P=0.714$ \\
\hline 70 & 12 & 8 & \\
\hline 80 & 7 & 8 & \\
\hline 90 & 3 & 2 & \\
\hline TACE & 15 & 14 & $P=0.499$ \\
\hline
\end{tabular}

AJCC American joint committee on cancer staging system, AFP $\alpha$ fetoprotein, KPS Karnofsky performance status, TACE transarterial chemoembolization

Fig. 2). Groups were compared using the Chi-square test; there was no difference among the two groups $(P=0.8707)$. The reaction degree was grade 1 or 2 and was all relieved after symptomatic treatment. All adverse reactions mainly occurred within 2 weeks following IRE, and only a few patients had fever following NK cell immunotherapy.

\section{Changes in Karnofsky Performance Status}

The pre-treatment KPS of the IRE group and IRE-NK group was $64.14 \pm 15.6$ and $65.96 \pm 17.8(P>0.05)$, respectively. The KPS was $74.13 \pm 11.2$ and $82.21 \pm 13.2$, respectively, 1 month post-treatment. The KPS of both groups was improved significantly posttreatment compared to pre-treatment. The KPS was significantly higher in the IRE-NK group at 1 month posttreatment (Fig. 4A, B).

\section{Changes in Tumor Biomarkers}

AFP (Fig. 5) and CTCs expression 1 month following treatment were lower in both groups $(P<0.05)$. There was no difference in biomarkers between the two groups prior to treatment $(P>0.05)$. However, 1 month post-treatment, CTCs (Fig. 6) expression was significantly lower in the IRE-NK $(P<0.05)$. No significant changes in CA 19-9 were observed in either group in this study.

\section{Discussion}

As a nonthermal ablation and minimally invasive therapy, IRE has brought therapeutic promise in unresectable PLC. Its high complete ablation rate and long recurrence-free period not affected by blood flow absorption makes it a powerful clinical tool. However, there were still a number of recurring patients in prior studies. [23-25] Immunosuppression in patients with PLC is an important factor leading to poor prognosis, recurrence, and metastasis. $[26,27]$ Murine studies have suggested that IRE therapy has a greater therapeutic effect in immunocompetent patients compared to those lacking a complete immune system [28]. Immunotherapy represents a promising new approach in the treatment of cancers [29]. However, immunotherapy alone cannot effectively prevent the progression of tumor, and it is difficult to counteract large tumor load. In recent years, multiple studies confirmed that the combination of local ablation treatments with immune stimulation appears to be a robust approach since immunotherapy can effectively target tumor cells of blood circulation that cannot be controlled by local ablation [21, 30].

As the first line of defense against tumor in human body, NK cells have a good prospect in the field of immunotherapy because they do not need the stimulation of specific antigens. NK cells can recognize major histocompability complex class I (MHC-I) molecules on the cell surface by KIR, and the activation of NK is inhibited when the two molecules are combined. Therefore, NK cells cannot be activated unless the MHC antigen on the surface of tumor cells mutates or disappears. It is generally accepted that KIR/HLA-mismatched NK cells are more effective in the treatment of malignant tumors and do not provoke graft-versus-host response. Our previous research 

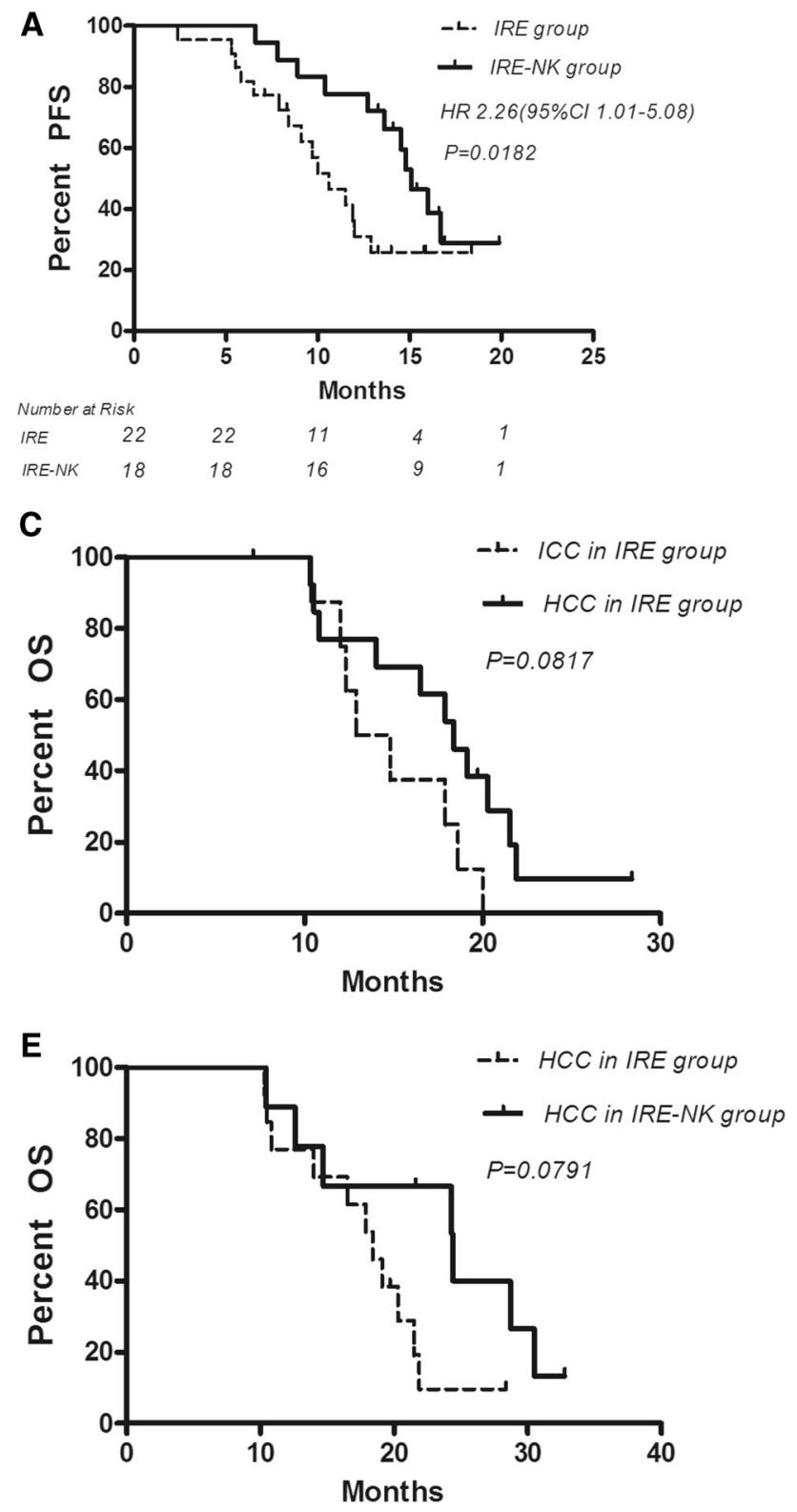

Fig. 2 A Progression-free survival shows that PFS in the IRE-NK group was significantly higher than in that of IRE group during follow-up period $(P<0.05)$. The median PFS in the IRE group was 10.6 and 15.1 months in the IRE-NK group. B Median OS for IRE group and IRE-NK group was 17.9 versus 23.2 months, with HR 2.25 (95\% CI 1.08-4.72). C OS of HCC and ICC in IRE group was not

Table 2 Clinical response 3 months post-treatment

\begin{tabular}{lllllll}
\hline Group & Total & CR & PR & SD & PD & RR (\%) \\
\hline IRE group & 22 & 1 & 14 & 6 & 1 & 68.2 \\
IRE-NK group & 18 & 3 & 13 & 2 & 0 & 88.9 \\
\hline
\end{tabular}

$C R$ complete response, $P R$ partial response, $S D$ stable disease, $P D$ progressive disease, $R R$ response rate
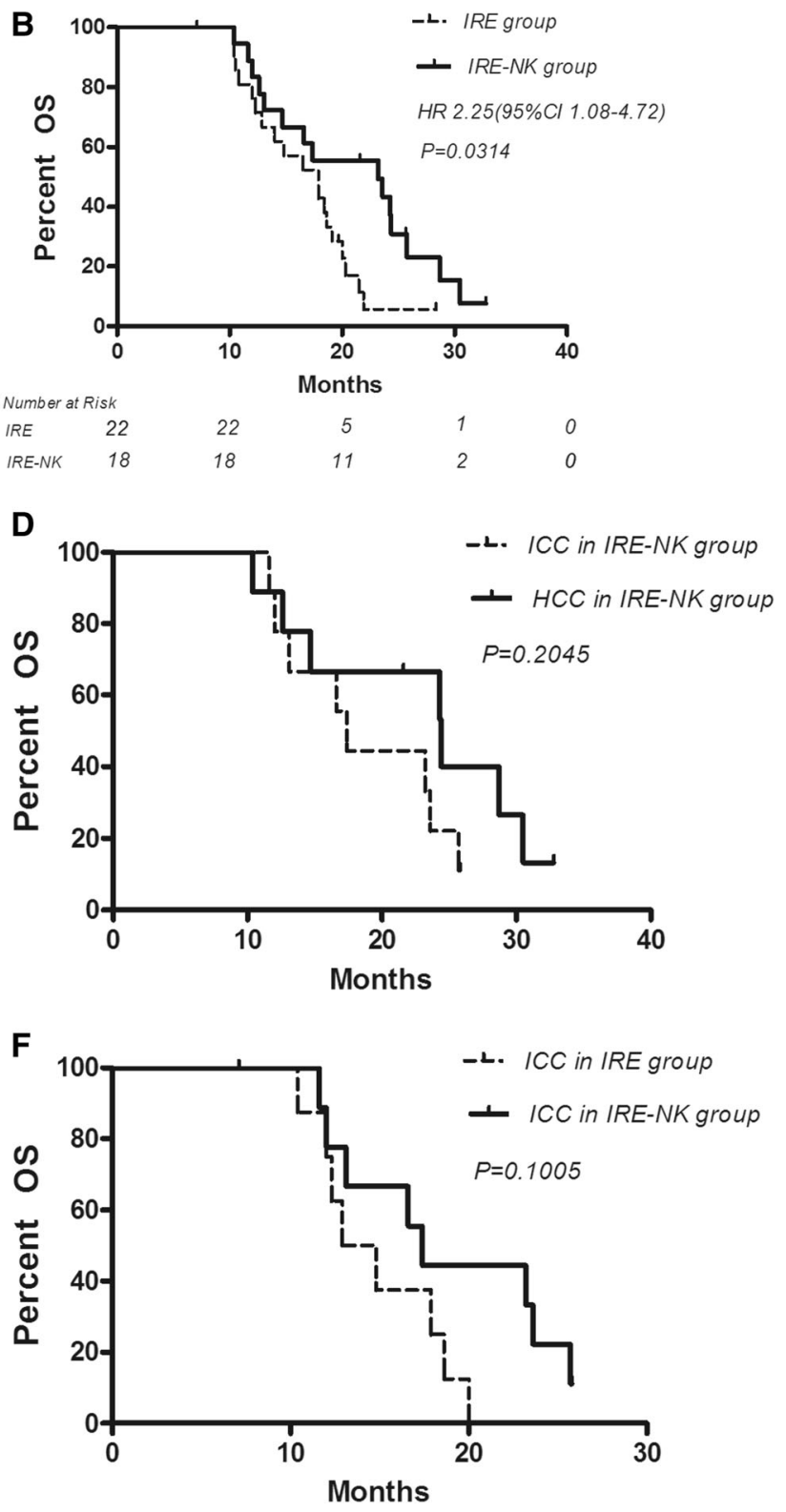

significantly different. D OS of HCC and ICC in IRE-NK group was not significantly different. E OS of HCC between IRE group and IRE-NK group was not significantly different. F OS of HCC between IRE group and IRE-NK group was not significantly different. $O S$ overall survival, $P F S$ progression-free survival, $H R$ hazard ratio

demonstrated that KIR-mismatched HANK cell immunotherapy is a safe therapy which improves the immune function of patients with liver cancer and reduces the rate of tumor metastasis and recurrence [19].

The pathophysiology of IRE is still not completely understood. In theory, the specific tumor cell antigen will not degrade from hyperthermia following the nonthermal IRE ablation; it may be well preserved as whole tumor cell vaccine. IRE ablation of tumor tissue retains the extracellular matrix, including vascular structure and lymphangion. 

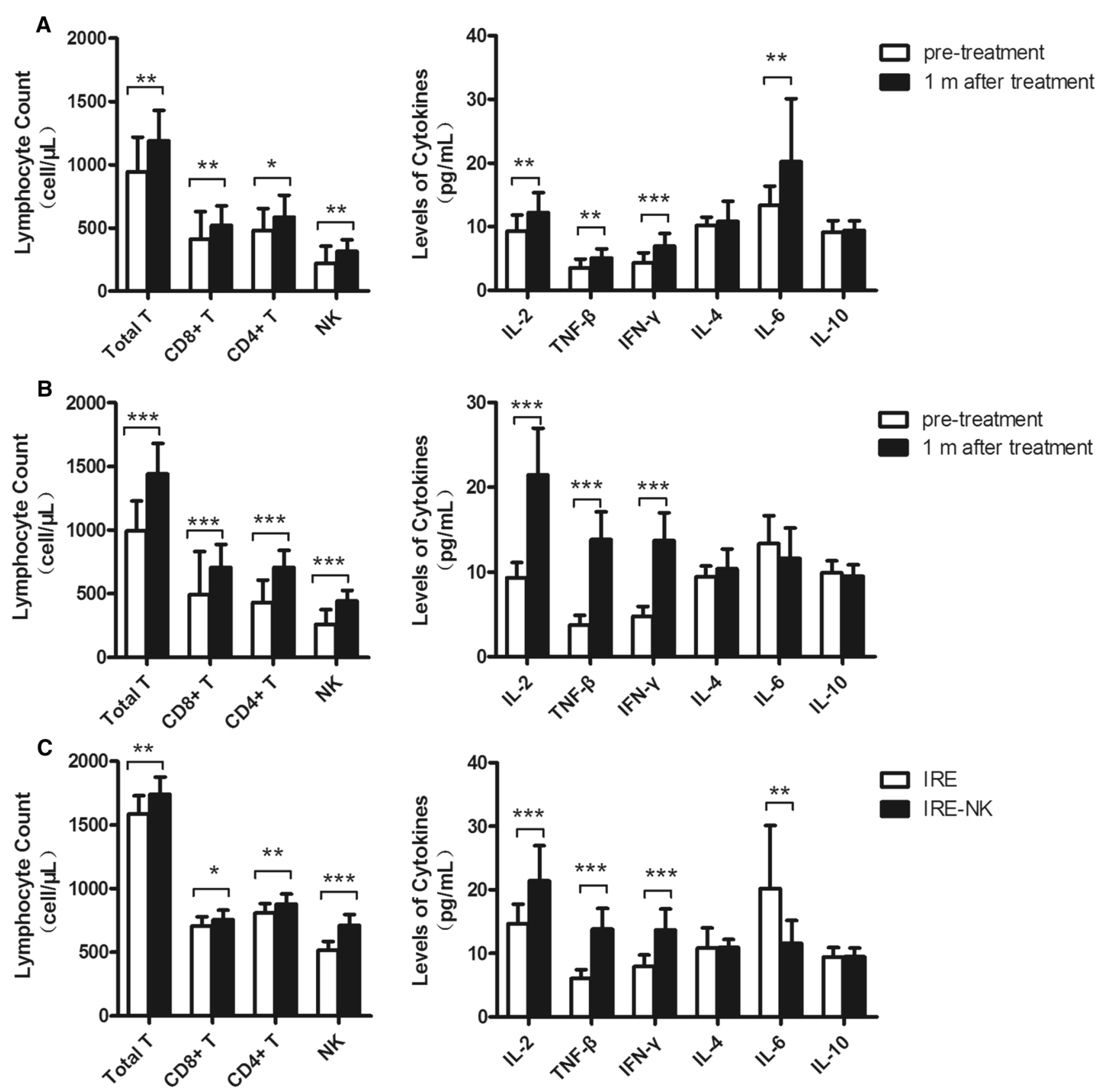

Fig. 3 A Changes in total T cells, NK cells, Th1 cytokines, and IL-6 were significant in the IRE group 1 month post-treatment; B Changes in total T cells, NK cells, and Th1 cytokines were significant in the IRE-NK group 1 month post-treatment; C At 1 month post-

Inactivated vascular endothelial cells can regenerate and revascularize following IRE, providing a structural basis for a variety of infiltrating immune cells. [13, 31, 32] Innate immune effector cells carry antigens from lymphatic vessels to lymph nodes, and then, activating anti-tumor $\mathrm{T}$ cell immune response in the draining lymph nodes, killer $\mathrm{T}$ cells can return to tumor site with circulation to eradicate residual tumor cells and prevent distant metastasis. Compared with the thermal ablation technique, IRE more treatment, total $\mathrm{T}$ cells, NK cells, and Th1 cytokines were significantly higher, while IL-6 was lower in the IRE-NK group compared to the IRE group. $* P<0.05, * * P<0.001$, $* * * P<0.0001$

effectively activates the immune function and induces a tumor immune response. In our study, the increase in Th1 cytokines and total $\mathrm{T}$ cells in the IRE group demonstrates that IRE treatment increases tumor susceptibility to host immunity. Furthermore, the increase in Th1 cytokines and total $\mathrm{T}$ cells in the combined therapy group was significantly higher than in the IRE treatment alone. The Th1 cytokines increased in this study, indicating that IRE combined with NK cell immunotherapy may shift the 

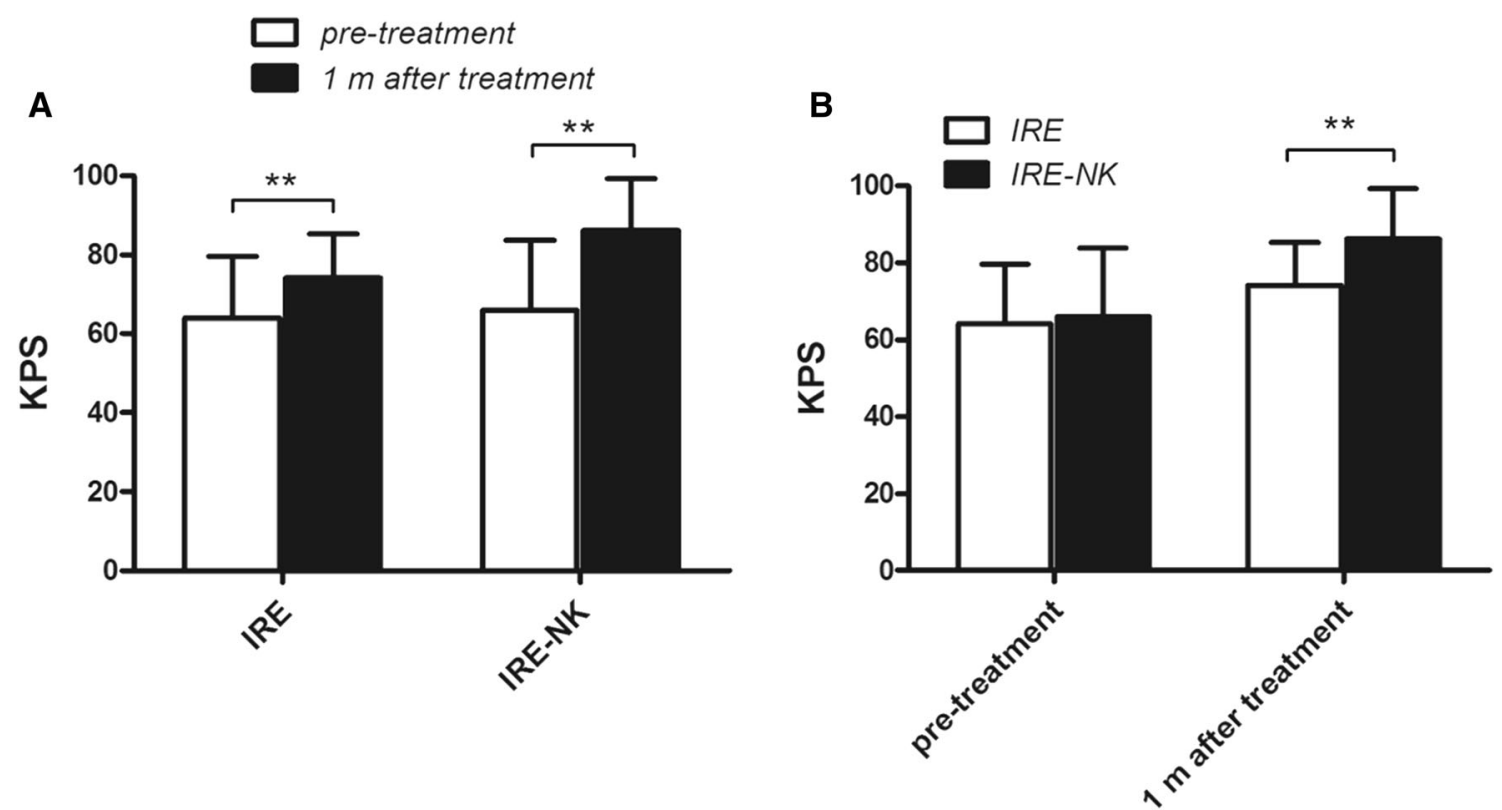

Fig. 4 A Changes in KPS in the IRE and IRE-NK groups. KPS was significantly increased in both groups 1 month post-treatment. B KPS was significantly increased in the IRE-NK group compared to the IRE group 1 month post-treatment. $* * P<0.01$

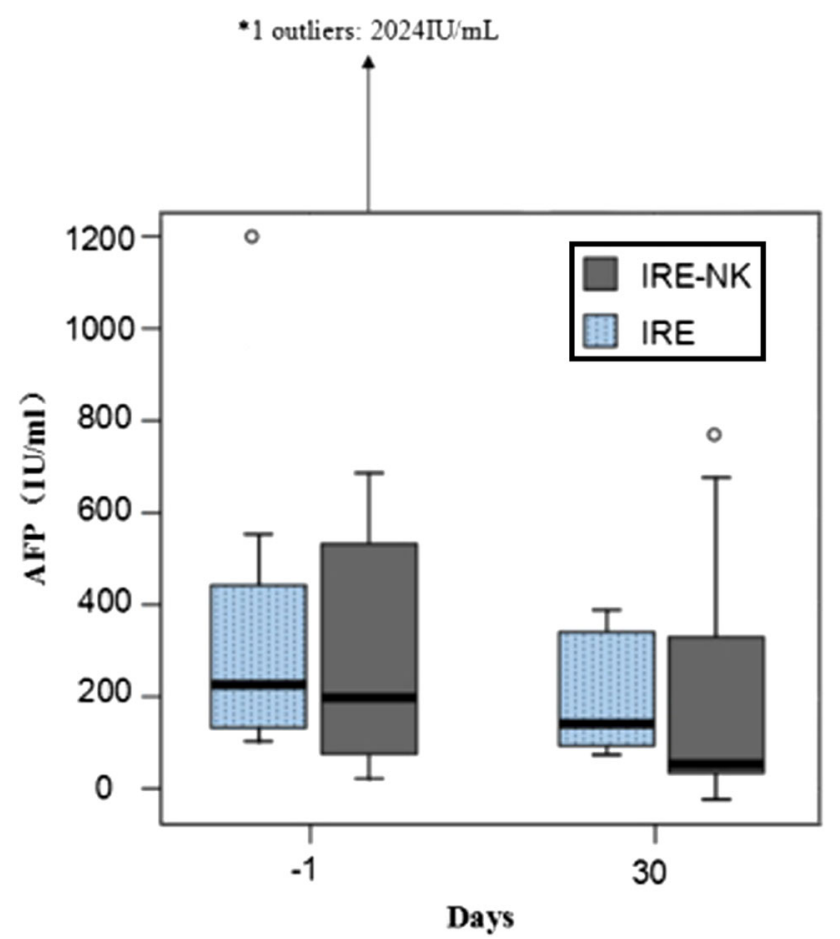

Fig. 5 Box-and-whisker plot shows AFP value before and after treatment. AFP was significantly decreased in both groups $(P<0.05)$

balance of Th1/Th2 and activate cellular immunity. No changes in Th2 cytokines were measured in the IRE-NK group; however, IL-6 in IRE group was significantly higher than prior to treatment. We speculate that it may be related to the immune activation mediated by damage-associated molecular pattern (DAMP). Mitochondrial DNA, high mobility group box-1(HMGB1), heat shock protein, and s100 proteins can be released by DAMP mode from necrotic cells. Then stimulate the corresponding TLR to produce cytokines including IL-6. Bulvik et al. [33] reported that peak IL-6 levels of IRE were three times higher than RF $6 \mathrm{~h}$ after liver ablation of female C57BL/6 mice. Their study suggests that IRE not only protects large blood vessels, but also preserves microcirculation and enhances the ability of cytokines included IL- 6 to enter the systemic circulation. As we know that IL-6 has potential tumorigenic ability, positively correlated with the progression of liver cancer, and negatively regulates the body's immunological function. In this study, No significant increase in IL-6 was observed in IRE-NK group, indicating that NK cell immunotherapy could regulate and improve the cellular immune response, positively regulate the immune system function and improve the anti-tumor effect. However, the specific mechanism of its role needs further study.

In this study, the response rate in 3 months reached 88.9 and $68.2 \%$ for IRE-NK and IRE, respectively. PFS and OS were significantly improved in the IRE-NK group, demonstrating the synergistic effect of these two therapies. Combined therapy seems superior to single IRE therapy in short-term efficacy. However, OS of HCC and ICC seems to have no significant differences $(P>0.05)$ in the interior group or inter-group. Considering that this study is a small sample of preliminary exploratory clinical study, the conclusions may be accidental and only for reference and need to be confirmed by larger clinical trials. In our previous study, the number of NK treatments also impacted on the 
Fig. 6 A Proportion analysis of CTCs prior to treatment;

B Proportion analysis of CTCs 1 month post-treatment; the number of CTCs was decreased significantly in the IRE $(\mathbf{C})$ and IRE-NK groups (D) 1 month post-treatment and is lower in the IRE-NK group $(\mathbf{E})$.

$* P<0.05, * * P<0.001$, $* * * P<0.0001$
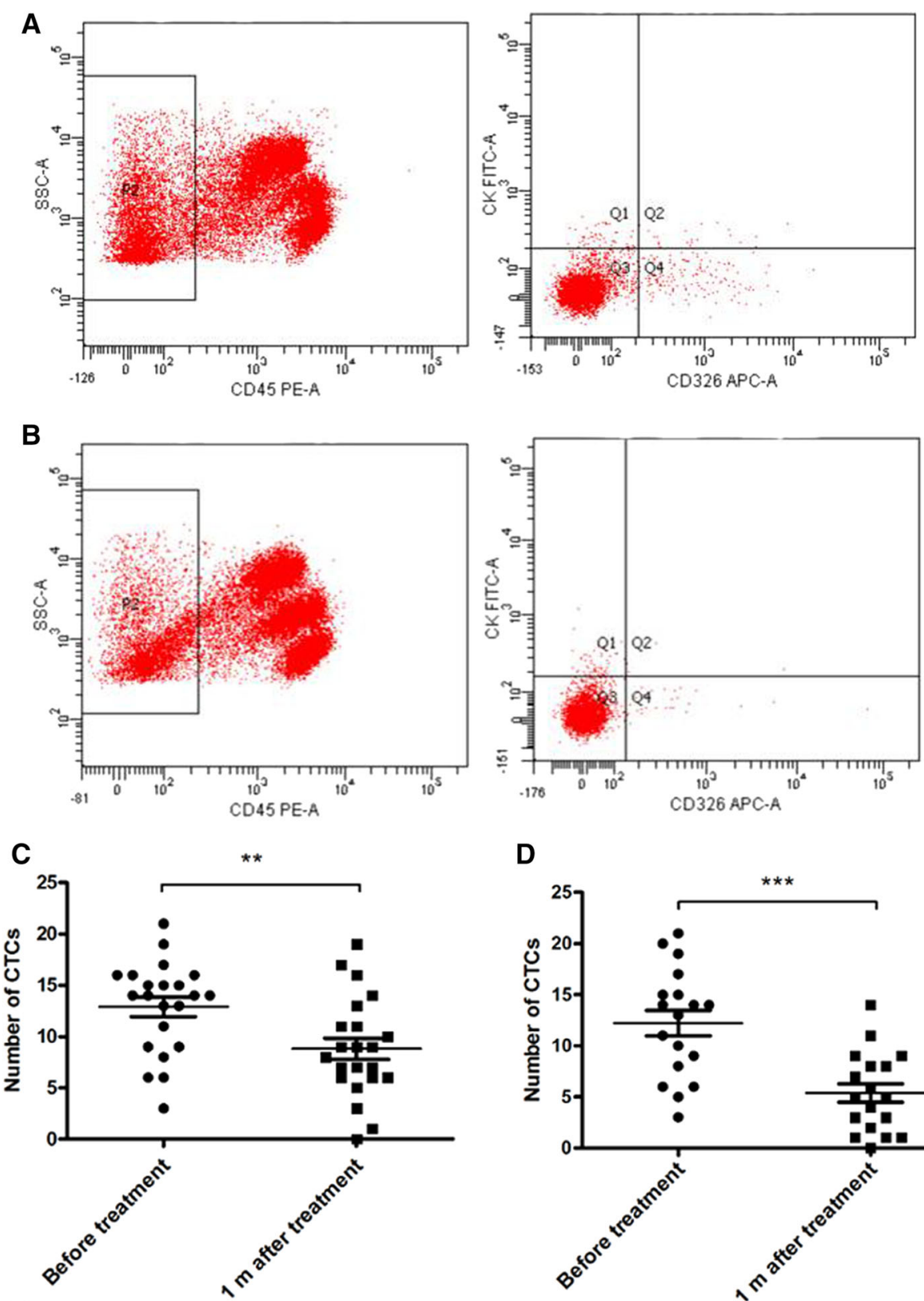

D

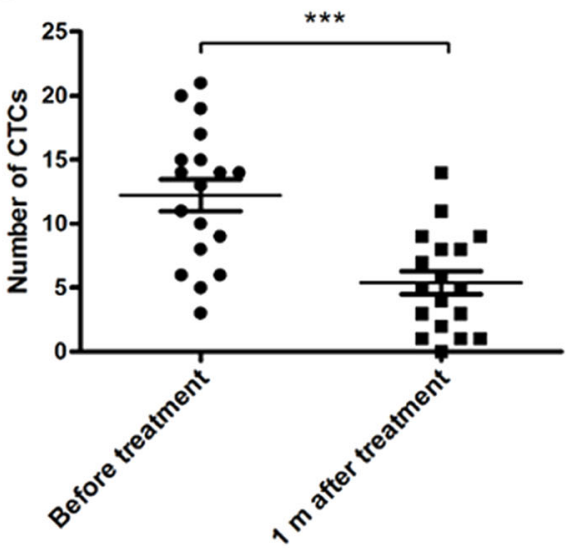

E

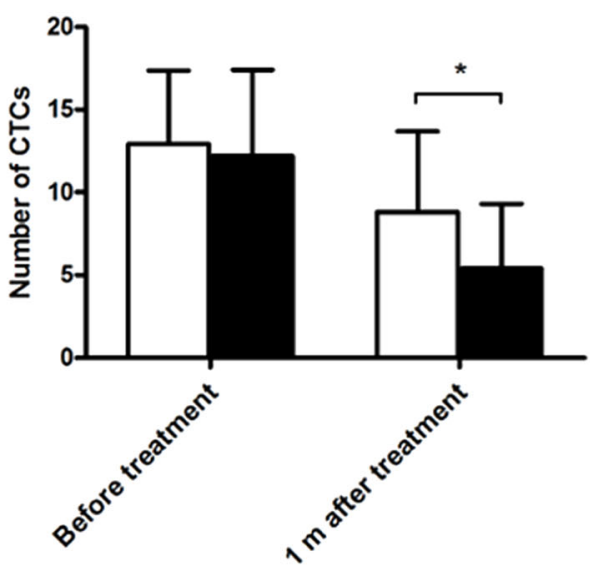


efficacy of the therapy; the PFS rate was significantly higher in patients who received more than 4 courses of NK cell therapy compared to those who received less than four [19]. Thus, the patients enrolled in this study were treated with 4 courses of NK cell therapy. However, the optimal number of treatment courses and the duration of treatment remain to be optimized.

The combination of NK cell immunotherapy immediately after IRE in the treatment of patients with unresectable PLC has not been reported. Its safety must be observed and verified since the immune status and development background of patients with PLC are complex. In this report, the side effects observed were not significant between both groups. The reaction degree was grade 1 or 2 , and all symptoms were relieved following symptomatic treatment. The adverse reactions mainly occurred within 2 weeks following IRE treatment, and only a few patients had fever following NK cell immunotherapy. This suggests that IRE combined with allogenic NK cell immunotherapy is well tolerated.

Biomarkers have an important role in the diagnosis, predicting the prognosis, and monitoring of the patients with PLC. AFP is one of the most common biomarkers for hepatocellular carcinoma (HCC). In our study, serum AFP significantly decreased in both groups and is lower in the NK-IRE group compared to the IRE group 1 month posttreatment. This indicates a better prognosis of the combination therapy. In addition, the CTC level is a biomarker that shedding from the primary tumor site and entering the peripheral blood circulation which positively correlated to tumor size [34]. We used CD326 as the positive selection while CD45 as the negative selection in the detection of peripheral blood CTCs to reduce the incidence of false negative. The decrease in CTCs observed in the present study may therefore reflect the improved efficacy of combination therapy. CA 19-9 is always used to monitor patients with ICC. However, there was no significant change in CA 19-9 in the present study. It appears that CA 19-9 is not sensitive to the outcome or prognosis of these treatments. However, the number of patients is too little, and some of the patients were already undergoing other therapies, such as surgery and TACE, before they were recruited. A larger sample of patients is needed to reach a more definitive conclusion.

In conclusion, this single-center prospective study has demonstrated the short-term safety and efficacy of IRE combined with allogenic NK cell immunotherapy for unresectable PLCs. However, the study has the following limitations: a small sample size and a short follow-up time. Therefore, long-term efficacy should be measured by extending the group of patients and increasing the period of follow-ups.
Acknowledgements We are grateful for the assistance of all patients and staff in Shenzhen Second People's Hospital and Fuda Cancer Hospital of Jinan University. This study was funded by grants from the Sanming Project of Medicine in Shenzhen [Grant No. SZSM201412009].

\section{Compliance with Ethical Standards}

Conflict of interest The authors declare that they have no conflict of interest.

Ethical Approval All procedures performed in studies involving human participants were in accordance with the ethical standards of the institutional and/or national research committee and with the 1964 Declaration of Helsinki and its later amendments or comparable ethical standards.

Informed Consent Informed consent was obtained from all individual participants included in the study.

Open Access This article is distributed under the terms of the Creative Commons Attribution 4.0 International License (http:// creativecommons.org/licenses/by/4.0/), which permits unrestricted use, distribution, and reproduction in any medium, provided you give appropriate credit to the original author(s) and the source, provide a link to the Creative Commons license, and indicate if changes were made.

\section{References}

1. Torre LA, Bray F, Siegel RL, Ferlay J, Lortet-Tieulent J, Jemal A. Global cancer statistics, 2012. CA Cancer J Clin. 2015;65(2):87-108. https://doi.org/10.3322/caac.21262.

2. Ferlay J, Soerjomataram I, Dikshit R, Eser S, Mathers C, Rebelo $\mathrm{M}$, et al. Cancer incidence and mortality worldwide: sources, methods and major patterns in GLOBOCAN 2012. Int J Cancer. 2015;136(5):E359-86. https://doi.org/10.1002/ijc.29210.

3. Lai EC, Fan ST, Lo CM, Chu KM, Liu CL, Wong J. Hepatic resection for hepatocellular carcinoma. An audit of 343 patients. Ann Surg. 1995;221(3):291-8.

4. Llovet JM, Real MI, Montana X, Planas R, Coll S, Aponte J, et al. Arterial embolisation or chemoembolisation versus symptomatic treatment in patients with unresectable hepatocellular carcinoma: a randomised controlled trial. Lancet. 2002;359(9319):1734-9. https://doi.org/10.1016/S0140-6736(02)08649-X.

5. Lo CM, Ngan H, Tso WK, Liu CL, Lam CM, Poon RT, et al. Randomized controlled trial of transarterial lipiodol chemoembolization for unresectable hepatocellular carcinoma. Hepatology. 2002;35(5):1164-71. https://doi.org/10.1053/jhep.2002. 33156.

6. Cheng AL, Kang YK, Chen Z, Tsao CJ, Qin S, Kim JS, et al. Efficacy and safety of sorafenib in patients in the Asia-Pacific region with advanced hepatocellular carcinoma: a phase III randomised, double-blind, placebo-controlled trial. Lancet Oncol. 2009;10(1):25-34. https://doi.org/10.1016/S1470-2045(08) 70285-7.

7. Sieghart W, Hucke F, Peck-Radosavljevic M. Transarterial chemoembolization: modalities, indication, and patient selection. J Hepatol. 2015;62(5):1187-95. https://doi.org/10.1016/j.jhep. 2015.02.010.

8. Llovet JM, Villanueva A, Lachenmayer A, Finn RS. Advances in targeted therapies for hepatocellular carcinoma in the genomic era. Nat Rev Clin Oncol. 2015;12(7):408-24. https://doi.org/10. 1038/nrclinonc.2015.103. 
9. Poulou LS, Botsa E, Thanou I, Ziakas PD, Thanos L. Percutaneous microwave ablation vs radiofrequency ablation in the treatment of hepatocellular carcinoma. World J Hepatol. 2015;7(8):1054-63. https://doi.org/10.4254/wjh.v7.i8.1054.

10. Guo Y, Zhang Y, Klein R, Nijm GM, Sahakian AV, Omary RA, et al. Irreversible electroporation therapy in the liver: longitudinal efficacy studies in a rat model of hepatocellular carcinoma. Cancer Res. 2010;70(4):1555-63. https://doi.org/10.1158/00085472.CAN-09-3067.

11. Rubinsky B, Onik G, Mikus P. Irreversible electroporation: a new ablation modality-clinical implications. Technol Cancer Res Treat. 2007;6(1):37-48. https://doi.org/10.1177/153303460700 600106.

12. Lu DS, Kee ST, Lee EW. Irreversible electroporation: ready for prime time? Tech Vasc Interv Radiol. 2013;16(4):277-86. https:// doi.org/10.1053/j.tvir.2013.08.010.

13. Maor E, Ivorra A, Rubinsky B. Non thermal irreversible electroporation: novel technology for vascular smooth muscle cells ablation. PLoS ONE. 2009;4(3):e4757. https://doi.org/10.1371/ journal.pone.0004757.

14. Dupuy DE, Aswad B, Ng T. Irreversible electroporation in a Swine lung model. Cardiovasc Interv Radiol. 2011;34(2):391-5. https://doi.org/10.1007/s00270-010-0091-9.

15. Schoellnast H, Monette S, Ezell PC, Deodhar A, Maybody M, Erinjeri JP, et al. Acute and subacute effects of irreversible electroporation on nerves: experimental study in a pig model. Radiology. 2011;260(2):421-7. https://doi.org/10.1148/radiol. 11103505.

16. Li X, Xu K, Li W, Qiu X, Ma B, Fan Q, et al. Immunologic response to tumor ablation with irreversible electroporation. PLoS ONE. 2012;7(11):e48749. https://doi.org/10.1371/journal. pone.0048749.

17. Niessen C, Beyer LP, Pregler B, Dollinger M, Trabold B, Schlitt $\mathrm{HJ}$, et al. Percutaneous ablation of hepatic tumors using irreversible electroporation: a prospective safety and midterm efficacy study in 34 Patients. J Vasc Interv Radiol. 2016;27(4):480-6. https://doi.org/10.1016/j.jvir.2015.12.025.

18. Ruggeri L, Capanni M, Urbani E, Perruccio K, Shlomchik WD, Tosti A, et al. Effectiveness of donor natural killer cell alloreactivity in mismatched hematopoietic transplants. Science. 2002;295(5562):2097-100. https://doi.org/10.1126/science.106 8440.

19. Qin Z, Chen J, Zeng J, Niu L, Xie S, Wang X, et al. Effect of NK cell immunotherapy on immune function in patients with hepatic carcinoma: a preliminary clinical study. Cancer Biol Ther. 2017;18(5):323-30. https://doi.org/10.1080/15384047.2017.1310 346.

20. Lin M, Liang S, Wang X, Liang Y, Zhang M, Chen J, et al. Shortterm clinical efficacy of percutaneous irreversible electroporation combined with allogeneic natural killer cell for treating metastatic pancreatic cancer. Immunol Lett. 2017;186:20-7. https:// doi.org/10.1016/j.imlet.2017.03.018.

21. Yu M, Li Z. Natural killer cells in hepatocellular carcinoma: current status and perspectives for future immunotherapeutic approaches. Front Med. 2017. https://doi.org/10.1007/s11684017-0546-3.
22. Appelbaum L, Ben-David E, Faroja M, Nissenbaum Y, Sosna J, Goldberg SN. Irreversible electroporation ablation: creation of large-volume ablation zones in in vivo porcine liver with fourelectrode arrays. Radiology. 2014;270(2):416-24. https://doi.org/ 10.1148/radiol.13130349.

23. Kingham TP, Karkar AM, D’Angelica MI, Allen PJ, Dematteo RP, Getrajdman GI, et al. Ablation of perivascular hepatic malignant tumors with irreversible electroporation. J Am Coll Surg. 2012;215(3):379-87. https://doi.org/10.1016/j.jamcollsurg. 2012.04.029.

24. Cheung W, Kavnoudias H, Roberts S, Szkandera B, Kemp W, Thomson KR. Irreversible electroporation for unresectable hepatocellular carcinoma: initial experience and review of safety and outcomes. Technol Cancer Res Treat. 2013;12(3):233-41. https:// doi.org/10.7785/tcrt.2012.500317.

25. Cannon R, Ellis S, Hayes D, Narayanan G, Martin RC 2nd. Safety and early efficacy of irreversible electroporation for hepatic tumors in proximity to vital structures. J Surg Oncol. 2013;107(5):544-9. https://doi.org/10.1002/jso.23280.

26. Brittenden J, Heys SD, Ross J, Eremin O. Natural killer cells and cancer. Cancer. 1996;77(7):1226-43.

27. Taketomi A, Shimada M, Shirabe K, Kajiyama K, Gion T, Sugimachi K. Natural killer cell activity in patients with hepatocellular carcinoma: a new prognostic indicator after hepatectomy. Cancer. 1998;83(1):58-63.

28. Neal RE 2nd, Rossmeisl JH Jr, Robertson JL, Arena CB, Davis EM, Singh RN, et al. Improved local and systemic anti-tumor efficacy for irreversible electroporation in immunocompetent versus immunodeficient mice. PLoS ONE. 2013;8(5):e64559. https://doi.org/10.1371/journal.pone.0064559.

29. Smyth MJ, Ngiow SF, Ribas A, Teng MW. Combination cancer immunotherapies tailored to the tumour microenvironment. Nat Rev Clin Oncol. 2016;13(3):143-58. https://doi.org/10.1038/ nrclinonc.2015.209.

30. Pardee AD, Butterfield LH. Immunotherapy of hepatocellular carcinoma: unique challenges and clinical opportunities. Oncoimmunology. 2012;1(1):48-55. https://doi.org/10.4161/ onci.1.1.18344.

31. Maor E, Ivorra A, Leor J, Rubinsky B. The effect of irreversible electroporation on blood vessels. Technol Cancer Res Treat. 2007;6(4):307-12. https://doi.org/10.1177/153303460700 600407.

32. Maor E, Ivorra A, Mitchell JJ, Rubinsky B. Vascular smooth muscle cells ablation with endovascular nonthermal irreversible electroporation. J Vasc Interv Radiol. 2010;21(11):1708-15. https://doi.org/10.1016/j.jvir.2010.06.024.

33. Bulvik BE, Rozenblum N, Gourevich S, Ahmed M, Andriyanov $\mathrm{AV}$, Galun E, et al. Irreversible electroporation versus radiofrequency ablation: a comparison of local and systemic effects in a small-animal model. Radiology. 2016;280(2):413-24. https://doi. org/10.1148/radiol.2015151166.

34. Liao Y, Wang SY, Meng XY, Yang J, Shi MJ, Liu HL, et al. Circulating tumor cells in breast cancer and its association with tumor clinicopathological characteristics: a meta-analysis. Med Oncol. 2014;31(12):343. https://doi.org/10.1007/s12032-0140343-7. 